\title{
SOME SERIES INVOLVING THE RIEMANN ZETA FUNCTION
}

T. M. APOSTOL

V. Ramaswami [1] has obtained a number of interesting recursion formulas which can be employed to obtain the analytic continuation of Riemann's zeta function $\zeta(s)$ over the whole $s$-plane. Denoting by $P_{n}(s)$ the polynomial defined by

$$
P_{0}(s)=1, \quad n ! P_{n}(s)=s(s+1) \cdots(s+n-1),
$$

three of Ramaswami's identities can be expressed as follows:

$$
\text { (3) } \zeta(s)\left(1-2^{-s}-3^{-s}-6^{-s}\right)=1+2 \sum_{n=1}^{\infty} P_{2 n}(s) \zeta(2 n+s) 6^{-2 n-s} \text {. }
$$

If we introduce the Hurwitz zeta function,

$$
\zeta(s, a)=\sum_{n=0}^{\infty}(n+a)^{-s} \quad(a>0, R(s)>1),
$$

so that $\zeta(s, 1)=\zeta(s)$, then we can easily obtain Ramaswami's results, as well as more general formulas, as consequences of some fundamental properties of $\zeta(s, a)$. Equation (1) is generalized by formula (8) below, whereas (2) and (3) are special instances of (13). Further general formulas are given in (9), (10), (11), and (15).

The basic properties of $\zeta(s, a)$ which we shall use are: the difference equation,

$$
\zeta(s, a)-\zeta(s, a+1)=a^{-s},
$$

the multiplication formula,

$$
\zeta(s, k a)=k^{-s} \sum_{n=0}^{k-1} \zeta(s, a+n / k)
$$

and the Taylor expansion of $\zeta(s, a+1)$ in the neighborhood of $a=0$,

Presented to the Society, June 20, 1953; received by the editors July 13, 1953. 


$$
\zeta(s, a+1)=\sum_{n=0}^{\infty}(-a)^{n} P_{n}(s) \zeta(n+s) .
$$

For fixed $s \neq 1$, the series in (7) converges absolutely for $|a|<1$. The expansion (7) follows at once from the fact that

$$
\partial \zeta(s, a) / \partial a=-s \zeta(s+1, a),
$$

whereas (5) and (6) can be established from the definition (4). The Hurwitz zeta function exists as an analytic function in the whole $s$-plane except for a simple pole at $s=1$ and formulas (5), (6), and (7) are valid for all $s$.

The basic idea which we use is the following. We apply (7) to various values of $a$ taken from some set $A$, where the set $A$ is chosen so that the sum

$$
\sum_{a \in A} \zeta(s, a+1)
$$

can be simplified. The simplification is effected by means of (5) and (6). For instance, if we take $a=-h / k, 0 \leqq h \leqq k-1$, and sum on $h$ we obtain

$$
\sum_{h=0}^{k-1} \zeta(s, 1-h / k)=\sum_{n=0}^{\infty} P_{n}(s) \zeta(n+s) k^{-n} \sum_{h=0}^{k-1} h^{n} .
$$

But by (6) we have

$$
\sum_{h=0}^{k-1} \zeta(s, 1-h / k)=\sum_{n=0}^{k-1} \zeta\left(s, \frac{1}{k}+\frac{n}{k}\right)=k^{s} \zeta(s),
$$

and hence we obtain the identity

$$
\zeta(s)\left(1-k^{1-s}\right)=\sum_{n=1}^{\infty} \frac{P_{n}(s) \zeta(n+s)}{k^{n+s}} \frac{B_{n+1}(k)-B_{n+1}}{n+1},
$$

where $B_{n}(x)$ is the $n$th Bernoulli polynomial and $B_{n}=B_{n}(0)$ is the $n$th Bernoulli number (in the notation of Nörlund [2]). When $k=2$, (8) reduces to (1).

If we now take $a=h / k, 1 \leqq h \leqq k-1$ in (7), sum on $h$ and take into account (5), we are led to the similar formula

$$
\begin{aligned}
\zeta(s)\left(1-k^{1-s}\right)= & \sum_{h=1}^{k-1} h^{-s} \\
& +\sum_{n=1}^{\infty}(-1)^{n} \frac{P_{n}(s) \zeta(n+s)}{k^{n+\imath}} \frac{B_{n+1}(k)-B_{n+1}}{n+1} .
\end{aligned}
$$


Adding (8) and (9) yields

$$
\zeta(s)\left(1-k^{1-s}\right)=\frac{1}{2} \sum_{n=1}^{k-1} h^{-s}+\sum_{n=1}^{\infty} \frac{P_{2 n}(s) \zeta(2 n+s)}{k^{2 n+s}} \frac{B_{2 n+1}(k)}{2 n+1},
$$

since $B_{2 n+1}=0$ for $n \geqq 1$. Subtracting (9) from (8) leads to a remarkable formula for the partial sums of the Dirichlet series for $\zeta(s)$, namely:

$$
\sum_{h=1}^{k-1} h^{-s}=\sum_{n=1}^{\infty} \frac{P_{2 n-1}(s) \zeta(2 n-1+s)}{k^{2 n-1+s}} \frac{B_{2 n}(k)-B_{2 n}}{n} .
$$

Incidentally, when $k=2$, formulas (10) and (11) reduce to equations (1) and (2) of Ramaswami's paper.

Next we apply (7) with $a=h / k, 1 \leqq h \leqq k / 2,(h, k)=1, k>2$, and sum on $h$ to obtain

$$
\begin{aligned}
\sum_{1 \leqq h \leqq k / 2,(h, k)=1} \zeta(s, 1+h / k) & \\
& =\sum_{n=0}^{\infty}(-1)^{n} P_{n}(s) \zeta(n+s) k^{-n} \sum_{1 \leqq h \leqq k / 2,(h, k)=1} h^{n} .
\end{aligned}
$$

Similarly we have

$$
\sum_{1 \leqq h \leqq k / 2,(h, k)=1} \zeta(s, 1-h / k)=\sum_{n=0}^{\infty} P_{n}(s) \zeta(n+s) k^{-n} \sum_{1 \leqq h \leqq k / 2,(h, k)=1} h^{n} .
$$

Adding these last two equations and using (5) gives us

$$
\begin{aligned}
& \sum_{1 \leqq h \leqq k,(h, k)=1} \zeta(s, h / k)=k^{s} \sum_{1 \leqq h \leqq k / 2,(h, k)=1} h^{-8} \\
& +2 \sum_{n=0}^{\infty} P_{2 n}(s) \zeta(2 n+s) k^{-2 n} \sum_{1 \leqq h \leqq k / 2,(h, k)=1} h^{2 n} .
\end{aligned}
$$

The sum on the left of (12) can be simplified by introducing the Möbius function $\mu(n)$ and writing

$$
\begin{aligned}
\sum_{1 \leqq h \leqq k,(h, k)=1} \zeta(s, h / k) & =\sum_{h=1}^{b} \sum_{d \mid(h, k)} \mu(d) \zeta(s, h / k) \\
& =\sum_{h=1}^{b} \sum_{d|h, d| k} \mu(d) \zeta(s, h / k) \\
& =\sum_{d \mid k} \mu(d) \sum_{m=1}^{k / d} \zeta(s, m d / k)=k^{s} \zeta(s) \sum_{d \mid k} \mu(d) d^{-s}
\end{aligned}
$$

where we have used (6). 
Hence we can write (12) as follows:

$$
\zeta(s) \sum_{d \mid k} \mu(d) d^{-s}=\phi(-s, k)+2 \sum_{n=0}^{\infty} P_{2 n}(s) \zeta(2 n+s) k^{-2 n-s} \phi(2 n, k),
$$

where $\phi(\alpha, k)=\sum_{1 \leqq h \leqq k / 2,(h, k)=1} h^{\alpha}$ is the sum of the $\alpha$ th powers of those integers not exceeding $k / 2$ which are relatively prime to $k$. When $k$ has the values $k=3,4$, or 6 , then $\phi(\alpha, k)$ becomes identically 1 and (13) simplifies considerably. Formulas (2) and (3) are the cases $k=3$ and $k=6$.

Finally, we use (7) with $a=h / k, 1 \leqq h \leqq k / 2, k \geqq 2$, and sum on $h$ to obtain

$$
\sum_{1 \leqq h \leqq k / 2} \zeta(s, 1+h / k)=\sum_{n=0}^{\infty}(-1)^{n} P_{n}(s) \zeta(n+s) k^{-n} \sum_{1 \leqq h \leqq k / 2} h^{n} .
$$

Similarly we have

$$
\sum_{1 \leqq h \leqq k / 2} \zeta(s, 1-h / k)=\sum_{n=0}^{\infty} P_{n}(s) \zeta(n+s) k^{-n} \sum_{1 \leqq h \leqq k / 2} h^{n} .
$$

Adding these and using (5) gives us

$$
\begin{aligned}
\sum_{h=1}^{k} * \zeta(s, h / k)=k^{8} & \sum_{1 \leqq h \leqq k / 2} h^{-8} \\
& +2 \sum_{n=0}^{\infty} P_{2 n}(s) \zeta(2 n+s) k^{-2 n} \sum_{1 \leqq h \leqq k / 2} h^{2 n}
\end{aligned}
$$

where $\sum^{*}$ means that the term corresponding to $h=k / 2$ must be doubled when $k$ is even. By (6) we can write

$$
\sum_{h=1}^{k} \zeta(s, h / k)=k^{s} \zeta(s)+\frac{1+(-1)^{k}}{2} \zeta(s, 1 / 2),
$$

and if we denote by $f(\alpha, k)$ the sum

$$
f(\alpha, k)=\sum_{1 \leqq h \leqq k / 2} h^{\alpha},
$$

we can write (14) in the form

$$
\begin{aligned}
\zeta(s)+\frac{1+(-1)^{k}}{2 k^{s}} & \left(2^{s}-1\right) \zeta(s) \\
& =f(-s, k)+2 \sum_{n=0}^{\infty} P_{2 n}(s) \zeta(2 n+s) k^{-2 n-s} f(2 n, k) .
\end{aligned}
$$


This formula also reduces to equation (1) of Ramaswami's paper when $k=2$.

\section{BIBLIOGRAPHY}

1. V. Rasmaswami, Notes on Riemann's $\zeta$-function, J. London Math. Soc. vol. 9 (1934) pp. 165-169.

2. Niels Nörlund, Vorlesungen über Differenzenrechnung, Berlin, 1924.

California Institute of Technology

\section{ON THE ASYMPTOTIC DISTRIBUTION OF CERTAIN SUMS}

\section{N. J. FINE}

1. Introduction. Let $((t))=t-[t]-1 / 2$, the brackets denoting the greatest integer function. It has been shown by Kac $[2]^{1}$ that the distribution of the sum $N^{-1 / 2} \sum_{0 \leqq n<N}\left(\left(2^{n} t\right)\right)$ is asymptotically normal with mean 0 and variance $1 / 4$. In $[1, \S 8]$, we considered the sum $\sum_{0 \leqq n<N}\left(\left(2^{n} t-1 / 2\right)\right)$ and proved that it is bounded uniformly in $t$ and $N$. It seemed of interest, therefore, to study the behavior of

$$
S_{N}(f ; t)=N^{-1 / 2} \sum_{n=0}^{N-1} f\left(2^{n} t\right)
$$

for

$$
f(t)=((t-\beta)), \quad 0 \leqq \beta<1 .
$$

It is proved in $\$ 2$ that the distribution is asymptotically normal, with mean 0 and variance

$$
\sigma^{2}(\beta)=\sum_{n=1}^{\infty} 2^{-n}\left(\left(\beta-\beta_{n}\right)\right)^{2},
$$

where $\beta_{n}$ denotes the fractional part of $2^{n} \beta$. Thus $\beta=1 / 2$ is the only case of zero variance. For this case we determine the limiting distribution of $N^{1 / 2} S_{N}(f ; t)$.

In $\S 3$ we consider $\gamma_{N}(t ; \alpha, \beta)$, the relative frequency with which $t_{n}=2^{n} t-\left[2^{n} t\right], 0 \leqq n<N$, falls in a given interval $(\alpha, \beta)$. Here, again, the distribution is asymptotically normal, and an explicit formula

Presented to the International Congress of Mathematicians, September 4, 1950; received by the editors August 5, 1953.

${ }^{1}$ Numbers in brackets refer to the bibliography at the end of the paper. 